\title{
La confiance dans la microfinance : le rôle de la proximité de langue
}

\author{
Cornelia Caseau \\ Groupe ESC Dijon-Bourgogne \\ Kouakou Kouamé \\ Université Alassane Ouattara Bouaké, Côte d'Ivoire
}

\section{INTRODUCTION}

\section{L'étude permettra enfin de démontrer qu'une communication intégrant le plurilinguisme constitue un facteur de confiance.}

Le concept de la confiance suscite encore aujourd'hui de nombreux débats dans le domaine bancaire et financier où il est étudié en lien avec la question de l'anticipation/dissipation du risque. Phénomène psychologique permettant avant tout la réduction de l'incertitude, la confiance se conçoit désormais comme un levier de succès pour les entreprises, voire une valeur centrale. Bruna et Deluzet (2014: 61) rappellent son importance managériale en ces termes : "Aux fondements d'un renouvellement $d u$ pacte social d'entreprise se place le rétablissement de la confiance $\gg$. Ce constat expliquerait aujourd'hui l'intérêt suscité par les nombreuses études portant sur l'identification et la description des déterminants et indicateurs de mesure de la confiance dans les entreprises (Kadem et Chetchoua-Kamyap, 2014). Mais la question de la confiance dans le domaine de la microfinance peut-elle s'accommoder de la langue, celle-ci s'analysant alors comme un facteur conditionnant sa consolidation dans ce milieu? Telle est la question à laquelle cet article tente de répondre.

Dans un premier temps, l'étude de la littérature permettra de dégager la nécessité du lien dans la transaction banque-client, reposant sur la consolidation de la confiance. On découvrira une nouvelle approche du discours commercial bancaire fondé sur une langue de proximité dans un contexte multiculturel, exemplifiée avec le cas autrichien étudié par Caseau et Bonescu (2014).

Dans un deuxième temps, l'article s'intéressera à la dimension stratégique du plurilinguisme à l'éclairage des expériences de la RLB NÖ-Wien et de la microfinance ivoirienne face à la barrière linguistique.

L'étude permettra enfin de démontrer qu'une communication intégrant le plurilinguisme constitue un facteur de confiance et dès lors une orientation féconde sur le plan managérial notamment pour la microfinance ivoirienne, mettant ainsi en exergue d'une façon plus ouverte l'importance de la compétence linguistique comme valeur économique d'une part, et comme avantage concurrentiel, d'autre part.

\section{CONFIANCE, PROXIMITÉ ${ }^{1}$ ET LIEN LINGUISTIQUE : UN LIEN NÉCESSAIRE DANS LES TRANSACTIONS BANQUE-CLIENT?}

Perçu aujourd'hui par certains comme une nécessité sociale, le service bancaire est au cœur des préoccupations managériales et fait également l'objet de recherches en marketing, en sciences sociales, etc. Dans sa conception karpikienne, la prestation de service bancaire est marquée par l'incertitude qui porte sur sa qualité, c'est-à-dire sur l'adéquation de la prestation aux besoins des différents acteurs. Dès lors, elle pose la question de son adaptation au contexte, voire celle de sa personnalisation. Sur la base de cette conception, des chercheurs comme Gloukoviezoff (2004) et celui de Gloukoviezoff et Lazarus (2005), Gloukoviezoff (2004), Caseau et Bonescu (2014), vont analyser le langage bancaire accessible à tous comme un facteur de rentabilité. Mais, c'est avec le récent travail de Caseau et Bonescu (2014) qu'on peut véritablement parler d'étude à visée linguis- 
tique qui se déploie désormais dans le champ du marketing bancaire. Étudiant les stratégies ethnomarketing développées par la Raiffeisenbank de Vienne, Caseau et Bonescu montrent comment les discours commerciaux et relationnels fondés sur une rhétorique de proximité affectent positivement l'attitude d'une clientèle d'immigrés originaires de Turquie et de l'ex-Yougoslavie vis-à-vis de cette banque. Elles définissent alors la proximité de langue comme le fait de s'adresser au client dans sa langue.

En Côte d'Ivoire, certaines IMFs (Le Crédit du Nord de Bouaké, la Coopérative d'Épargne et de Crédit (COOPEC) de Nimbo-Bouaké, par exemple), conscientes de l'idéologie qui fonde la microfinance et du rôle de l'usage des langues locales dans la captation et la fidélisation de la clientèle vulnérable linguistiquement, développent actuellement un modèle de communication plurilingue proactif pour rendre encore plus efficace le service auprès de cette clientèle. C'est un modèle qui s'appuie sur les ressorts suivants pour fonctionner : la méthode d'appui sur le personnel. Cette méthode consiste à recourir à un membre du personnel de la banque qui maîtrise la langue du client; autre méthode, l'appui sur un accompagnateur (le client se fait accompagner par quelqu'un, généralement un parent instruit, qui lui relaie l'information en langue locale, mais aussi qui joue les autres rôles liés à la communication écrite (remplir un chèque, signature, etc.); enfin l'appui sur la clientèle. Cette dernière stratégie de communication qui est rarement sollicitée parce qu'étant en déphasage avec l'un des esprits qui caractérisent l'éthique bancaire (la discrétion et la confidentialité des opérations), consiste à recourir

\section{MÉTHODE DE RECUEIL DES DONNÉES}

\section{Cette étude exploratoire s'appuie sur une} collecte de données obtenues grâce à des informations recueillies auprès de la

Raiffeisenbank, une banque coopérative autrichienne et des IMFs ivoiriennes.

Cette étude exploratoire portant sur le rôle du contact personnel conseiller-client et de la langue comme vecteur de confiance s'appuie sur une collecte de données obtenues grâce à des informa- dans le hall de l'agence à un autre client pour relayer l'information en langue locale au client qui a des difficultés de compréhension.

Ainsi, pour contribuer au débat sur le rôle de la confiance dans la consolidation de l'image des IMFs, nous cherchons à savoir en quoi la proximité des liens linguistiques, un concept qui participe de la dialectique de près et de loin dans les interactions entre les IMFs et leur clientèle, peut être vue comme vecteur de confiance et levier managérial.

Ces faits nous ont permis de formuler des hypothèses suivantes :

Hypothèse 1: le fait de concevoir une communication fondée sur une endogénéisation des moyens linguistiques à l'endroit des cibles subissant involontairement ou non la reculturation ${ }^{2}$ est une démarche stratégique qui peut renforcer la confiance de ces cibles à l'endroit de l'entreprise.

Hypothèse 2 : Parler la langue du client est un gage d'une image de marque valorisante et d'une identité forte pour l'entreprise, car la langue rapproche l'entreprise du client, elle constitue ainsi un facteur de confiance en soi.

Pour contribuer au débat sur le rôle de la confiance dans la consolidation de l'image des IMFs, nous cherchons à savoir en quoi la proximité des liens linguistiques [...] peut être vue comme vecteur de confiance et levier managérial. tions recueillies auprès de la Raiffeisenbank, une banque coopérative autrichienne et des IMFs ivoiriennes. Dans le cadre autrichien, nous avons analysé des supports écrits ainsi que les entretiens menés essentiellement au siège et dans différentes filiales de la RLB NÖ-Wien de Vienne pratiquant l'ethno-banking. Ces entretiens ont été réalisés entre 2011 et 2014 et ont donné un aperçu du quotidien linguistique multiculturel de ces filiales et de l'importance de la proximité et de la relation interpersonnelle (Berry, 1983). Un entretien réalisé 
avec Manuel Bräuhofer, directeur de l'Agence de Marketing Brainworker, présentant et expliquant les initiatives ethno-marketing de son entreprise pour la RLB NÖ-Wien a complété les enquêtes. Un entretien avec la gérante de WirtschaftfürIntegration, une association fondée sur l'initiative de la Raiffeisenbank a permis d'approfondir notre recherche.

Les entretiens ont généralement duré une heure et ont été enregistrés et transcrits.

Ils étaient basés principalement sur les questions suivantes :

- Les raisons de l'intérêt de la banque RLB NÖ-Wien pour les communautés d'immigrants;

- Les mesures entreprises pour gagner leur confiance;

- La proximité/le contact avec les clients
- Les particularités des échanges avec les communautés d'immigrants;

- Le rôle de la langue et de la culture dans les échanges;

- La sensibilisation/formation des collaborateurs de la banque.

Pour comprendre le fonctionnement de la langue dans le modèle de communication de la microfinance ivoirienne, nous avons mené en 2012 et en 2014 une série d'observations dans sept (7) agences d'IMFs évoluant à l'intérieur du pays (voir liste en annexe), suivies d'entretiens auprès de dirigeants, personnels de guichet et de clients de ces microfinances. Un questionnaire leur a été aussi soumis.

La taille de l'échantillon par effectif et par pourcentage des personnes interrogées se présente comme suit :

\begin{tabular}{|l|c|c|}
\hline Modalités & $\begin{array}{c}\text { Nombre de personnes } \\
\text { interrogées }\end{array}$ & Fréquence \\
\hline Dirigeants & 9 & $30 \%$ \\
\hline Personnels de guichet & 9 & $30 \%$ \\
\hline Clients analphabètes & 12 & $40 \%$ \\
\hline TOTAL & $\mathbf{3 0}$ & $\mathbf{1 0 0} \%$ \\
\hline
\end{tabular}

Enregistrés et transcrits par la suite, la durée de ces entretiens était généralement de trente à quarantecinq minutes. Les questions portaient principalement sur :

- La confiance

- Les mesures entreprises pour gagner la confiance

- La proximité/le contact avec les clients
- Les particularités des échanges avec les clients analphabètes et de faible niveau en langue française

- L'attitude de leur entreprise sur le problème d'accessibilité linguistique

- Le ressenti des clients analphabètes et semi-alphabètes sur les deux modes de communication, à savoir :

$\circ$ en français exclusivement

$\circ$ en langues locales

\section{RÉSULTATS}

\subsection{Les vecteurs de la confiance}

\subsubsection{Proximité de langue et confiance dans la} communication de la RLB NÖ-Wien

Grâce à leur attachement régional, les banques Raiffeisen ont toujours eu une relation très étroite avec leurs clients, plaçant l'homme au centre de leurs préoccupations (Rapport d'activité 2011). Selon l'esprit de son fondateur allemand F. W. Raiffeisen (1818-1888), un des initiateurs du mou- vement coopératif, considéré également comme précurseur de l'idée moderne de la microfinance, le groupe bancaire Raiffeisen continue à se référer à la vocation de la responsabilité sociale et sociétale ${ }^{3}$. Conformément à cet objectif, les ethno-filiales sont situées au cœur des arrondissements viennois à forte concentration d'immigrants. À côté des conseillers autrichiens germanophones, la banque embauche des collaborateurs d'origine turque ou des pays d'ex-Yougoslavie. Dix des 72 filiales viennoises 
(qui sont d'ailleurs indiquées sur le site de la banque), travaillent avec plus de 30 collaborateurs au service des communautés étrangères et reçoivent leurs clients dans leur langue maternelle. Le contact avec les clients est si étroit, que les employés de banque sont même reconnus par leurs clients dans la rue et au marché du quartier.

Dès lors, quand le conseiller de banque dispose aussi bien des compétences linguistiques nécessaires, que des connaissances de la culture de l'interlocuteur (définie par la banque comme langue derrière la langue), la confiance s'établit plus facilement.

\section{Quand le conseiller de banque dispose aussi bien des compétences linguistiques nécessaires, que des connaissances de $l a$ culture de l'interlocuteur, la confiance s'établit plus facilement.}

\subsubsection{Les vecteurs de confiance de la microfinance ivoirienne}

La communication microfinancière ivoirienne identifie plusieurs éléments comme vecteurs de la confiance. Ce sont le temps, la religion, l'écoute, le respect de la parole donnée et la langue. Faute d'espace pour des raisons d'édition, nous insisterons sur le facteur langue, qui ressort clairement des entretiens comme étant la pierre angulaire :

«Etre à l'écoute du client, lui donner toutes les garanties sur son compte, répondre à ses besoins ou exigences permettent de bâtir une relation de confiance. Mais le client a plus confiance en sa banque si l'agent qui est en face de lui s'exprime dans sa langue. J'en ai fait l'expérience dans au moins trois ou quatre villages » (INTER 1).

La langue est créatrice de lien, elle favorise surtout la sociabilité. Forte de ce pouvoir, elle permet de dissiper dans une relation à visées mercantilistes où l'incertitude est de mise, les susceptibilités que l'usager-client nourrit à l'encontre d'un agentbancaire sous le prétexte qu'il est issu d'une autre communauté linguistique :

«Je suis ressortissant de Béoumi (une ville du centre de la Côte d'Ivoire). Bien avant que je ne vienne à Tiébissou, les ressor- tissants de Béoumi ici à Tiébissou animaient un réseau communautaire d'entre-aide. Mais ils gardaient leur fortune à la maison. Mais lorsque je me suis présenté à eux, lors de mon adhésion à leur réseau, comme ressortissant de Béoumi, ils se sont précipités vers la RCMEC pour garder leur argent. Ils exprimaient leur réaction à travers cet adage baoulét $^{4}$ : «SE $\supset$ sifuc o do nu, do di'à wo » (ou littéralement en français : «Là où vous avez un proche, il vous a toujours apporté sa protection) » (INTER 1).

Un autre agent renchérit en ces termes :

"Quand j'étais à Abengourou (Abengourou est une ville du sud-est de la Côte d'Ivoire), j'étais agent de crédit. Malgré les explications du gérant (un Abey ${ }^{5}$ ), les clients malinké $e^{6}$ qui ne parlent pas français préfèrent m'attendre. C'est quand je viens et que je leur donne des explications en malinké qu'ils se sentent rassurés. Contents qu'ils sont après mes explications, ils repartent chez eux » (INTER 2).

Si la langue, dans son fonctionnement social comme attribut identitaire et culturel, renforce les liens entre l'entreprise et ses clients les plus vulnérables linguistiquement et/ou économiquement, il ressort aussi de nos entretiens que sa fonction d'instrument de communication dont la mise en signe facilite la compréhension entre les protagonistes, consolide ce lien en permettant à l'agent d'être plus proche du client. La contribution de la langue à la construction d'une politique managériale de proximité se révèle aussi lorsque l'agent parle la même langue que lui ou adapte son discours à son niveau de langue :

«Pour nous, être plus près du client, c'est s'inscrire dans sa peau, se mettre à sa place. Dans les institutions classiques lorsque vous arrivez, l'agent ne dispose pas de temps pour vous. Alors que chez nous, lorsque vous arrivez, on vous fait assoir. On vous explique ce que vous ne comprenez pas. Si vous avez un niveau de français bas, on ramène notre langage à ce niveau. Si vous ne comprenez. pas ou ne savez pas parler le français, on vous reçoit dans votre langue » (INTER 1). 
Le rôle de créateur de confiance de la langue entre l'entreprise et sa clientèle est aussi perceptible à travers le ressenti des clients analphabètes et semianalphabètes envers les agents de guichet, lorsque ceux-ci utilisent les langues locales pour les accueillir. En effet, sur les douze personnes interrogées, huit, soit $67 \%$ d'entre elles, déclarent faire plus confiance aux agents qui ne les reçoivent pas en français. Aussi, $58 \%$ considèrent de leur côté que l'usage des langues locales les rapproche des agents qui les reçoivent en communiquant avec elles dans ces langues. Pour cette catégorie de clients, ce comportement linguistique conditionne encore plus leur rapport à ces agents, qu'ils voient désormais comme étant des frères ou sœurs, au sens premier de ces termes.

Si les langues locales constituent une corde sensible sur laquelle surfent les agents des organisations visitées pour développer une communication de proximité comme gage de confiance entre eux et les clients linguistiquement vulnérables, ils sont aussi d'avis que compte tenu de la prédominance de l'oralité, cette entreprise n'est possible que grâce à d'autres éléments constitutifs de la confiance que sont l'écoute, l'accès facile au service et la parole donnée.

\section{Le rôle de créateur de confiance de la langue entre l'entreprise et sa clientèle est aussi perceptible à travers le ressenti des clients analphabètes et semi-analphabètes envers les agents de guichet.}

\subsection{Les implications managériales de la proximité de langue sur la microfinance ivoirienne}

La relation bancaire est par essence une relation qui repose sur la confiance. Gadrey (cf. Gloukoviezoff et Tinel (2004)) la définit comme une relation de coproduction au sens de la division technique du travail entre les acteurs. Pour cerner les implications managériales du recours aux langues autochtones, nous nous sommes intéressés particulièrement au rôle de cette politique d'endogénéisation linguistique au niveau des interactions client-entreprise. Ainsi, nous avons cherché à comprendre le ressenti que les entreprises visitées ont de cette politique ainsi que celui des clients envers les entreprises, lorsque celles-ci utilisent ou non le français pour les accueillir.

\subsubsection{Entre client et entreprise}

Les statistiques montrent aussi que l'indogénéisation linguistique donne une image de marque valorisante et une identité forte à l'entreprise qui la pratique.

Alors que l'usage exclusif du français est perçu comme un frein à la communication entre l'entreprise et les clients vulnérables, il ressort de nos entretiens que le recours aux langues locales facilite la compréhension mutuelle.

Les statistiques montrent aussi que l'indogénéisation linguistique donne une image de marque valorisante et une identité forte à l'entreprise qui la pratique. Ainsi, 66,70\% des clients interrogés se déclarent très contents d'être servis en langues locales, contre $16,70 \%$ qui n'ont pas d'opinion et $16,70 \%$ qui se disent mécontents du recours aux langues locales. Concernant leur avis sur la continuation de politique d'indigénisation de la communication des IMFs, la moitié des clients se déclare favorable, soit $50 \%$, alors que $41,70 \%$ de ces clients restent sans avis; $8,30 \%$ ne voient pas l'utilité de cette pratique.

\subsubsection{Côté entreprise}

Selon la définition que Gloukoviezoff (2006) lui en donne, en référence à Gadrey, la relation bancaire est une pratique langagière où les rôles sont distribués. Ainsi, l'usage exclusif du français rend le client qui ne parle pas cette langue de l'entreprise, insatisfait dans la relation. Comme conséquence marketing, c'est parfois le refus de traiter avec cet agent qu'on ne comprend pas, et face auquel on ne peut s'exprimer, faute de partager un langage commun.

Certains employés voient ces comportements comme une menace pour l'entreprise et préviennent leurs supérieurs immédiats quant au danger que ce problème fait courir.

D'autres employés font par contre dans le cadre de leur rapport annuel ou de fin de service des recommandations pour remédier à ce problème. 
En général, dirigeants, personnel de guichet et clients analphabètes partagent tous un avis très favorable sur la question d'une politique de communication mixte alternant le français et les langues autochtones. Pour preuve, 77,80\% des dirigeants interrogés sont d'accord pour une formation linguistique des agents. Quand 55,60\% d'entre eux envisagent d'opter désormais une politique de recrutement du personnel de guichet

\section{DISCUSSIONS}

\section{L'étude révèle que l'usage des langues nationales dans sa politique de communication rapproche davantage la microfinance de la clientèle analphabète ou de faible niveau d'instruction.}

$\mathrm{Au}$ terme de cette analyse, rappelons les deux hypothèses initialement émises, à savoir d'une part, que la conception d'une communication fondée sur la proximité linguistique comprise comme démarche stratégique permet de renforcer la relation banque-client et d'autre part, que la maitrise des langues locales dans l'échange avec le client valorise l'image de l'entreprise.

L'expérience autrichienne de la RLB NÖ-Wien et la description de la situation de la microfinance en Côte d'Ivoire permettent ainsi de mettre en évidence non seulement l'importance du concept de proximité dans la communication reposant sur le plurilinguisme de l'entreprise bancaire, en général et microfinancière, en particulier, mais encore la constitution d'un véritable capital de confiance devenu un atout dans la performance économique.

La prise en compte du quotidien linguistique multiculturel et l'importance donnée à la proximité dans la relation avec le client issu des communautés d'immigrants ont été érigées en véritable stratégie managériale par la banque autrichienne. Donnant à cet établissement bancaire un réel avantage concurrentiel dans le prolongement néanmoins de l'idéal de son fondateur.

Dans le contexte ivoirien, comme dans la plupart des pays africains, les principales institutions de fondé sur des compétences linguistiques et culturelles.

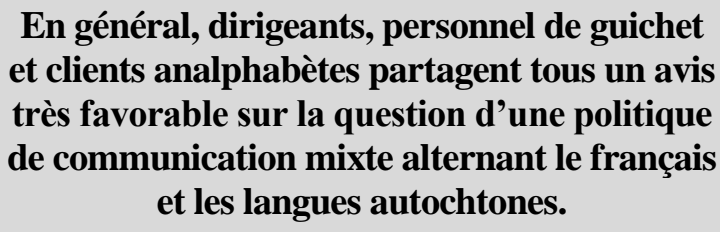

financement de l'économie qu'a connues le pays au lendemain de son indépendance sont les banques et les compagnies d'assurance. Or, le fonctionnement de celles-ci obéit à des exigences qui ne sont pas très souvent de nature à favoriser leur accès aux agents économiques ayant un niveau d'instruction relativement bas et/ou analphabètes.

Apparues depuis les années 1970, les IMF ont pour mission de résoudre ce problème social. Par définition, la microfinance, fille de l'économie sociale et solidaire se caractérise par son autonomie de gestion par rapport à l'État, mais également par rapport à la conception traditionnelle de l'économie. En somme, de par son fonctionnement démocratique, l'émergence de la microfinance est un défi à l'économie néo-libérale. Pour relever ce défi, voire celui de la compétitivité, il semble incontournable que les IMF ivoiriennes aient une connaissance fine de l'environnement dans lequel elles interviennent. Cette attitude pourrait leur permettre d'identifier et de prendre en compte les facteurs pouvant servir de création de valeur économique nouvelle et bien sûr d'avantages concurrentiels.

À ce niveau, l'étude a relevé que la microfinance ivoirienne évolue sans considération aucune de la variable langues nationales dans ses mécanismes d'interactions alors que cela semble être une évidence. En outre, l'étude révèle que l'usage des langues nationales dans sa politique de communication rapproche davantage la microfinance de la clientèle analphabète ou de faible niveau d'instruction. Confirmant ainsi l'hypothèse du rôle d'invariant marketing des langues nationales. 
En faisant cette étude, nous n'avons nullement la prétention d'une vision deus ex machina de l'utilisation des langues nationales dans la gestion de la relation client des IMF ivoiriennes. Les IMF ont constamment besoin d'informations valables et fiables pour leurs politiques de développement, et spécialement pour leur politique de services où elles doivent prendre des décisions allant dans le sens de leur amélioration. Compte tenu de la spécificité linguistique de l'environnement marketing ivoirien, nous estimons que penser du devenir de la banque ne devrait plus relever du seul apanage des disciplines traditionnelles du management bancaire que sont le marketing, la publicité, la sociologie, l'anthropologie, l'économie, le droit, la psychologie...

\section{CONCLUSION : DE SERVET À RAIFFEISEN, OU LA (NOUVELLE) TRADUCTION LINGUISTIQUE DE LA CONFIANCE BANCAIRE, QUEL INTÉRÊT MANAGÉRIAL POUR LA MICROFINANCE IVOIRIENNE?}

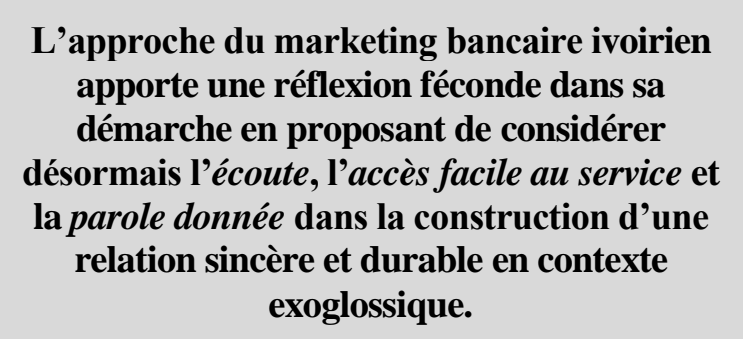

Des recherches antérieures ont montré qu'un échange personnalisé dans la langue maternelle suscite plus de confiance et crée une fidélisation émotionnelle. Dans ce travail, nous nous sommes fixés comme but de mettre en évidence les attentes du client à l'égard de son entreprise lorsque subsistent des barrières linguistiques, et le type de communication à mettre en place lorsqu'une entreprise est confrontée à une telle situation. Pour atteindre cet objectif, nous sommes partis de l'hypothèse qu'une communication intégrant le plurilinguisme serait un facteur gagnant, et que la microfinance ivoirienne, qui vit une crise de modèle de communication pour des raisons inhérentes à la situation sociolinguistique dans laquelle elle évolue, pourrait s'en inspirer pour assoir une communication source de confiance face à sa clientèle linguistiquement vulnérable.

Que retenir comme contributions et perspectives aux termes de notre étude?

Selon le National Center for Cultural Competence du Canada : «(La) compétence linguistique est la capacité d'une organisation et de son personnel de communiquer de façon efficace et de transmettre l'information d'une façon facilement compréhensible par des clientèles diversifiées, incluant les personnes ayant une connaissance limitée (ou non) de la langue de la majorité, ceux qui ont un faible degré de littératie ou qui sont illettrés. » (Aucoin, 2008: 9). En partant de cette idée, nous notons qu'au plan managérial, la microfinance ivoirienne et la banque Raiffeisen ont développé des modèles de communication pour résoudre la question de la barrière linguistique face à leur clientèle potentielle. Cependant, le modèle ivoirien pèche à bien des égards en insuffisances qui pourraient être corrigées par le modèle autrichien. Alors que le fonctionnement du modèle ivoirien obéit à une logique de sérendipité qui le met en déphasage avec l'esprit et la démarche marketing, le modèle de la banque Raiffeisen est l'œuvre d'une action réfléchie et bien pensée. Entre autres actions des recherches sur la préférence linguistique des clients, une politique de recrutement d'un personnel fondée sur la compétence culturelle et linguistique et des ateliers de formation du personnel culturel et linguistique.

Généralement, pour définir la confiance, les recherches antérieures, fondées sur la conceptualisation euro-centrée, se focalisent sur l'expertise, la sincérité et la bienveillance, la sympathie. De son côté, l'approche du marketing bancaire ivoirien apporte une réflexion féconde dans sa démarche en proposant de considérer désormais l'écoute, l'accès facile au service et la parole donnée dans la construction d'une relation sincère et durable en contexte exoglossique. Du coup, elle place la prise en compte des langues minoritaires au cœur de la problématique de l'efficacité communicationnelle lorsque la langue de la majorité peut s'analyser comme obstacle. Car si parmi les trois antécédents identifiés, nous nous appuyons sur l'écoute et la parole donnée pour illustrer notre 
argumentation, cet intérêt du rôle des langues locales dans le questionnement de l'efficacité de la communication bancaire en contexte ivoirien est dû au fait que les opérations bancaires découlent d'un processus actif et interactionniste. Dès lors, cette hypothèse amène à se demander comment les protagonistes engagés dans ce processus peuvent s'écouter et se comprendre, s'ils ne disposent pas d'une langue commune pour se parler. Puisque généralement, on s'accorde à dire que la meilleure communication est obtenue lorsque les protagonistes parlent la même langue. Concernant la parole donnée, Servet l'a identifiée comme élément constitutif de la confiance dans les sociétés où l'oralité domine. Or la sincérité, selon Fodor (1983) suppose d'inférer de ce qu'on entend dire et de comprendre à ce que le monde est. En partant de ce postulat, on voit que cet autre élément constitutif de la confiance pose le problème du partage de langue entre protagonistes puisqu'ici aussi on ne peut juger la valeur d'une promesse que si elle est émise dans une langue qu'on maîtrise.

Bref, d'aucuns nous rétorqueront que ces concepts dénotent d'un tour de langue dans la mesure où une analyse fine de ces antécédents montre que la parole donnée correspondrait à la notion classique de sincérité. Quant à l'écoute, elle pourrait être assimilée à la bienveillance. Pour autant, cette nouvelle approche de la confiance ouvre d'autres voies de recherche pour comprendre et stimuler l'importance de la compétence linguistique quand la barrière linguistique devient un problème managérial pour l'entreprise.

\section{BIBLIOGRAPHIE}

Aucoin, L. (2008), Compétences linguistiques et culturelles des organisations de santé. Analyse cri-tique de la littérature, Disponible sur http://www. rssfe.on.ca/files/uploads/rssfefiles/rapport_aucoin_ssf_ version_finale2008.pdf.

Berry, L.L. (1983), Relationship Marketing, Emerging perspectives on Services Marketing, Ed. Berry, Shostack\&Upah, AMA, 1983, pp. 25-28.

Bräuhofer, M. \& Yadollah-Farsani, R. (2011), Ethnomarketing in Österreich. Praxishandbuch. HolzhausenVerlag. Wien.

Bruna, M. G. \&Deluzet, M. (2014), (Re)tissage de la confiance et nouveau pacte social : défis et conditions de déploiement des politiques du capital humain, Question(s) de Management?, nº?, pp. 57-72, Éditions EMS.
Caseau, C. \&Bonescu, M. (2014), Les enjeux de l'ethnomarketing dans le secteur bancaire : le cas d'une banque autrichienne, Gérer et Comprendre, ${ }^{\circ} 118$, pp. 30-40.

Darpy, D. \& Silhouette-Dercourt, V. (2008), La reculturation dans le processus de consommation : approche par le carré sémiotique d'ici/de là-bas, http://basepub.dauphine.fr/xmlui/handle/123456789/278 Dupuy, C. \& Torre, A. (2005), Local clusters, trust, confidence and proximity, Christos

P., Sugden R., Wilson J. (eds.) Clusters and Globalisation: The Development of Urban and

Regional Economies Edward Elgar, Cheltenham UK, Northampton USA.

Fodor, J. (1983), The modularity of my mind, MIT Press. Gilly, J.P. \& Pecqueur, B. (1997), Régulation et territoire, une approche des dynamiques institutionnelles locales, Deuxièmes Journées de la Proximité, Proximités et coordination économique, Lyon.

Gloukoviezoff, G. (2006), Clients modestes et services bancaires: «les exclus de l'intérieur», Lazuech, G. \&Moulévrier, P., Contribution à une sociologie des conduites économiques, Paris : L'Harmattan, pp. 25-32.

Gloukoviezoff, G. (2004b), De la bancarisation de masse à l'exclusion bancaire puis sociale, Revue Française des Affaires Sociales, n³-2004, Paris: La documentation française, pp. 11-38.

Gloukoviezoff, G. (2004a), La médiation de proximité, une solution à l'exclusion bancaire ?, Recherche Sociale, Paris : FORS-Recherche Sociale, pp.62-77.

Gloukoviezoff, G. \&Lazarus, J. (2005),La relation bancaire avec la clientèle : des particuliers : revue de littérature, Volet $1:$ La relation de service dans la banque. Disponible surhttps://gloukoviezoff. files.wordpress. com/2009/01/relation_bancaire_volet1_integral.pdf.

Gloukoviezoff, G. \&Tinel, B. (2004), Un client peut en cacher un autre. L'accueil du public par La Poste et une mairie d'arrondissement, Rapport de Recherche, Disponible sur http://gloukoviezoff. files.wordpress. com/2009/01/rapport-final-2005.pdf, Consulté le 22/03/2012.

Hall, E. T. (1963), A system for the Notation of ProxemicBehaviour, Americain Anthropologist, vol. 65, pp. 1003-1026.

Herault-Fournier et al. (2014), Diagnostiquer la proximité perçue en vente directe de produits alimentaires, version finale à paraître dans Décisions Marketing $\mathrm{n}^{\circ} 73$.

Kadem, E. \&ChetchouaKamyap, D. (2014), La confiance organisationnelle dans une firme multinationale: cas de Corly Cameroun S.A., Question(s) de Management?, $\mathrm{n}^{\circ} 8$ ?, pp. 93-95, Editions EMS.

Karpik, L. (1989), L'économie de la qualité, Revue française de sociologie, XXX-2, pp. 187-210.

Lindskold, S. (1978), Trust development, the GRIT proposal, and the effects of conciliatory acts on 
conflict and cooperation, Psychological Bulletin, 85 772-93.

Moulins, J.-L. et al. (2012), De la qualité de service à la fidélité des clients : une investigation sur le secteur bancaire au Vietnam, Economies et sociétés,vol. 46, nº4, pp. 815-836.

Olivier, A. \& de Maricourt, R. (1990), Pratique marketing en Afrique, EDICEF/AUPELF.

Ouarda, C. \&Zghal, M. (2008),Les facteurs expli-catifs de l'utilisation des technologies bancaires libre-service, Disponible sur http://www.iemniae. univnantes.fr/50926888/0/fiche__pagelibre/.

Revenu Canada (1997), Besoins d'information et stratégies des Canadiens ayant un faible niveau d'alphabétisme, Rapport final présenté à Revenu Canada, Disponible sur http://fr.copian.ca/biblio/ recherche/ revcan/cogem2f.pdf.

Robien, M. (de) (1997), Banques, votre pub m'intéresse!, Banque Editeur.

Rotter, J.B. (1980), Interpersonal trust, trust-worthiness, and gullibility, American Psychologist, vol. 35, pp. 1-7.

Simmel, G. (1991), Secret et sociétés secrètes, Strasbourg, Circé.

Talbot, D. (2009), L’approche par la proximité : quelques hypothèses et éléments de définition, Communication à la XVIII' ${ }^{\mathrm{e}}$ conférence de l'AIMS, Grenoble, Disponible sur http://www.strategie-aims.com/events/conferences/ 3-xviiieme-conference-de-l-aims/communications/200lapproche-par-la-proximite-quelques-hypotheses-etelements-de-definitions/download.

Torrès, O. (2011), Proxémies financières des PME. Les effets collatéraux de la financiarisation des banques, Revue française de gestion, $\mathrm{n}^{\circ} 213$, Hermès, Lavoisier, pp. 189-204.

Torrès, O. (2002), Essai de conceptualisation proxémique de la petitesse des entreprises, $\sigma^{e}$ Congrès International Francophone PME (CIFPME 2002), HEC Montréal, Dis-ponible sur http://www.oliviertorres.net/travaux/pdf/ ot17cifpme02.pdf.

Vallée, P.-H. \&Mackaay, E. (2006), La confiance. Sa nature et son rôle dans le commerce électronique, LexElectronica, vol. $11 \mathrm{n}^{\circ} 2$ (Automne / Fall 2006), Disponible sur http://www.lex-electronica.org/articles/ v11-2/vallee_mackaay.htm.

Zolliger, M. \& Lamarque, E. (2009), Marketing et stratégie de la banque, Dunod.

\section{NOTES}

${ }^{1}$ D'après les travaux de Hall (1963), repris par Abraham Moles et Elisabeth Rohmer en 1973, la proximité se définit comme « une tendance naturelle de l'homme à privilégier ce qui est proche de lui au détriment de ce qui s'en éloigne » (Torrès, 2011).

${ }^{2} \mathrm{La}$ reculturation est un retour à la culture d'origine qui passe par la consommation (Darpy\& Silhouette-Dercourt, 2008). C'est un processus qui peut être subi. Dans ce cas, les individus déjà acculturés, confrontés à l'absence de produits adaptés à leur besoin, se tournent vers des produits ou marques issus de la culture d'origine. La reculturation peut aussi être volontaire, le consommateur exprimant dans ces conditions le besoin qu'il a de revenir vers les produits, les marques ou lieux de vente liés à sa culture d'origine. En Europe, la reculturation s'observe au sein des groupes minoritaires issus de l'immigration et en interaction avec la culture majoritaire, lorsque les individus issus de ces groupes redécouvrent leur culture d'origine à travers la consommation de produits ou de services ethniquement marqués. ${ }^{3}$ Pour faciliter la lecture du texte, les citations allemandes des entretiens sont traduites et mises en italiques.

${ }^{4}$ Le baoulé est une langue-ethnique, parlée au centre de la Côte d'Ivoire.

${ }^{5}$ L'abey est une langue-ethnique parlée au sud de la Côte d'Ivoire, dans la région d'Agboville.

${ }^{6}$ Le malinké est une langue-ethnique, parlée au nord de la Côte d'Ivoire. 


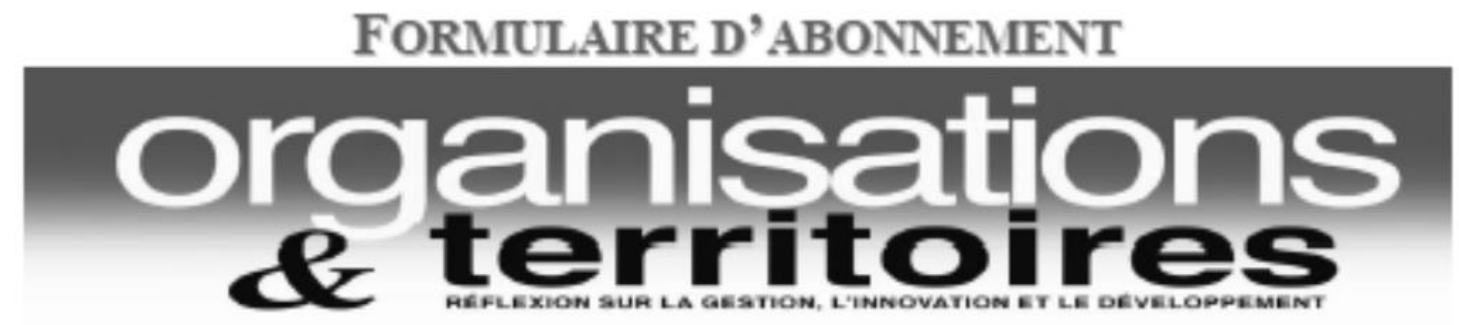

Je désire m'abonner à la revue Organisations et territoires et je joins un chèque au montant de : s

Nouvel abonné $(\sqrt{ }): \square$ Renouvellement $(\sqrt{ }): \square$ Date :
Abonnement institutionnel
(6 numéros)
$103,48 \$$ (Toutes taxes incluses)
Abonnement individuel
(6 numéros)
$66,69 \$$ (Toutes taxes incluses)
Abonnement étudiant
(6 numéros)
$48,29 \$$
(Tx inc.) (Envoyer photocopie carte étud.)
Abonnement hors Canada
(6 numéros) $145,00 \$$
$(110 \$+35 \$$ frais de poste) (sans tass)

Tous les abonnements sont d'une durée de deux ans

- J'aimerais que mon abonnement débute avec le DERNIER numéro paru, soit :

Volume $\mathrm{N}^{\circ}$

- J'aimerais que mon abonnement débute avec le PROCHAIN numéro à paraître, soit :

Volume $\mathrm{N}^{\circ}$

Faites-nous parvenir vos coordonnées :

Nom :

Organisation :

Adresse:

Teléphone:

Telécopieur :

Courriel :

Adresse d'expédition :

Revue Organisations et territoires Université du Québec à Chicoutimi 555 , boulevard de l'Université, bureau H6-1360

Chicoutimi (Québec) CANADA G7H 2Bl

Téléphone : (418) 545-5011, poste 4530 Télécopieur : (418) 693-9072

Courriel : revueot $@$ uqac.ca Site Web : www.uqac.ca/revueot 